\title{
Determination of ultra-low milk fat content using dual-wavelength ultraviolet spectroscopy
}

\author{
Shiqiong Xiong, ${ }^{*}$ Benu Adhikari, $†$ Xiao Dong Chen, ${ }^{*}$ and Liming Che ${ }^{* 1}$ \\ *Department of Chemical and Biochemical Engineering, Xiamen University, Xiamen 361005, P. R. China \\ †School of Science, RMIT University, Melbourne, VIC 3083, Australia
}

\begin{abstract}
A precise determination of milk fat content in dairy products is of great practical importance. Several studies have reported methods to determine the milk fat content in commercial dairy products. However, very few of them validated or tested cases where the fat milk fat content was ultra-low. Here we present an approach to determine ultra-low milk fat content. This method involves extracting milk fat using $n$-heptane and determining its content using dual-wavelength UV spectroscopy. The results showed that milk fat at ultralow concentrations (i.e., as low as $0.148 \mathrm{~g} / \mathrm{L}$ ) could be extracted completely and quantified accurately using the proposed approach. This method is reproducible and sufficiently precise. We expect that this method can be adapted by industries to determine ultra-low concentration of fat in milk and other dairy products with confidence.
\end{abstract}

Key words: dual-wavelength, milk fat, UV spectroscopy

\section{INTRODUCTION}

Milk contains approximately $3.4 \%$ (wt) fat. Milk fat has the most complex fatty acid composition among edible fats. Over 400 individual fatty acids have been identified in milk fat so far, of which approximately 15 to 20 fatty acids make up $90 \%$ of the milk fat. The major fatty acids in milk fat are straight-chain fatty acids that are saturated or polyunsaturated (Milk Facts, 2015). More than 95\% of the milk fat is in the form of globules, ranging in diameter from 0.1 to $15 \mu \mathrm{m}$. It is well known that if milk is left to stand, fat globules will cream due to the difference in density between the dispersed fat and continuous aqueous phase (Fox, 2011).

Due to the importance of milk fat, several methods have been developed to determine its content in dairy

Received June 20, 2016.

Accepted August 18, 2016.

${ }^{1}$ Corresponding author: lmc@xmu.edu.cn products (El-Abassy et al., 2011). The dairy industry makes use of the methods developed especially for fat; for example, the Röse-Gottlieb, Gerber, and Babcock methods (Forcato et al., 2005).

The Röse-Gottlieb method extracts fat from milk repeatedly with organic solvent (i.e., diethyl ether or petroleum ether). The milk fat content is determined gravimetrically after removing the organic solvent via evaporation (Manirakiza et al., 2001). This method is simple and it does not require an expensive analytic instrument; however, it requires a large amount of sample and organic solvent to determine the fat content in dairy products when the fat content is relatively low. Due to the sensitivity limitation of the weighing system (e.g., balance) used, this method can give rise to large errors in measured milk fat content.

The Gerber method was developed and patented by Niklaus Gerber of Switzerland in 1891 (Badertscher et al., 2007). This method is simple, provides results rapidly, and is inexpensive. It is the standard method to determine fat content of dairy products in Europe. Specific Gerber tubes, or butyrometers, have been designed for different dairy products such as whole milk, skim milk, and cream. Nonfat solids in the sample are dissolved by adding concentrated sulfuric acid. Separation of fat from aqueous phase is accomplished by adding amyl alcohol followed by centrifugation. The fat content is read directly by using a calibrated butyrometer. Although the Gerber method is effective and reliable, several specialized apparatus, such as butyrometer, pipette, centrifuge, and water bath, are required to carry out the test (James, 1995). The Gerber method cannot be automated and involves a certain risk in handling concentrated sulfuric acid. The handling of the butyrometer also requires practical skill, which has a negative effect on the robustness of the method (Badertscher et al., 2007).

The Babcock method, which is more common in the United States, is similar to the Gerber method. It differs from the Gerber method in the nature of the glassware used and in the specification of caliper used to measure the length of the fat column (James, 1995). It suffers 
the same disadvantages of the Gerber method. In nonindustrial laboratories, densitometric (Badertscher et al., 2007), chromatographic (Spanos et al., 1995; Povolo and Contarini, 2009), dielectric (Zhu et al., 2015), laser light scattering (Xin et al., 2006), and spectrometric (Carl, 1991; Tsenkova et al., 1999; Forcato et al., 2005; Bernuy et al., 2008; El-Abassy et al., 2011) technologies have been developed to determine the content of milk fat.

Badertscher et al. (2007) decomposed milk with hydrochloric acid and then extracted fat from the milk with hexane. The content of fat was then obtained by measuring the density of the extracts with a densitometer based on the principle of resonant frequency U-tube oscillation. Fat contents of milk, cream, and cheese can be determined conveniently using this method. The values of milk fat content obtained from this method did not differ significantly from those obtained by gravimetric reference methods.

Attempts have been made to determine milk fat using chromatographic techniques, such as GC and HPLC (Spanos et al., 1995; Povolo and Contarini, 2009). One of the drawbacks of GC is that milk fat is not stable and it can degrade when heated to gasification; HPLC, on the other hand, can handle thermally labile compounds, such as milk fat. However, the sophisticated hardware and software used in HPLC make it rather expensive when compared with other analytical tools. Additionally, HPLC requires specialized columns, which are expensive and have a relatively short operating life.

Zhu et al. (2015) determined the fat content in cow milk making use of dielectric properties of milk. A linear relationship between the dielectric properties and the fat content was reported by Zhu et al (2015). However, the linear coefficient required for determination was low when the temperature increased above $37^{\circ} \mathrm{C}$, which limited the applicability of this method.

Laser light-scattering technology has also been used to determine the fat content in fresh milk. The ratio of scattered to transmitted light intensity, which is known as scattered-transmitted ratio, was found to increase almost linearly with the increase in fat content. However, the dependence of scattered-transmitted ratio on fat content is sensitive to the conditions of sample preparation, such as the pressure and temperature of homogenization. Another disadvantage of this method is that it requires a sophisticated instrument (Xin et al., 2006).

El-Abassy et al. (2011) demonstrated the capability of visible Raman spectroscopy as a rapid technique for direct determination of milk fat. The visible Raman spectroscopy relies on the fact that change in the relative Raman band intensity correlates well with the over- all change in milk fat content. For the purpose of in-line monitoring of milk fat, no extraction and separation steps are employed. The signals of other substances in milk, such as proteins and sugars, contribute to background during the test, which significantly limits the sensitivity of this method.

Near-infrared absorption spectroscopy also serves as a rapid technique for direct determination of fat in homogenized (Carl, 1991) or unhomogenized (Tsenkova et al., 1999) liquid milk. The disadvantage of nearinfrared absorption spectroscopy is that it suffers from water interference, as milk contains about $87.5 \%$ water, which absorbs infrared light strongly (El-Abassy et al., 2011).

Forcato et al. (2005) presented a fast, cost-effective, and highly sensitive method for the determination of milk fat, which made use of UV-adsorbing properties of milk fat. The hydrophobic proteins and peptides, which interfere with UV determination, were precipitated by adding absolute ethanol. After centrifugation of the milk sample, the supernatant was transferred to a cuvette and the fat content was measured directly using a UV spectrophotometer. To minimize the use of organic solvent, Forcato et al. (2005) avoided the extraction of milk fat, which resulted in considerable error in the measured milk fat content.

All of the methods reviewed above were developed to determine the fat content in commercial dairy products, which have a relatively high fat content. The reliability or effectiveness of these methods in determination of ultra-low milk fat content has not yet been tested. The determination of ultra-low milk fat content is sometimes more important, for instance, during the cleaning of milk pipelines and dairy equipment. Therefore, the aim of this work was to develop an approach to determine ultra-low milk fat content more accurately. This work consisted of the extraction of milk fat with small amounts of organic solvent and the subsequent determination of the extracted milk fat using dualwavelength UV spectroscopy.

\section{MATERIALS AND METHODS}

\section{Materials}

Whole milk with a fat content of $37 \mathrm{~g} / \mathrm{L}$ came from Inner Mongolia Mengniu Dairy (Group) Co., Ltd. (Hohehot, China). The received milk sample was heated to $80^{\circ} \mathrm{C}$ and then homogenized using a microfluidizer (M110P, Microfluidics International Corporation, Newton, MA) at $30 \mathrm{MPa}$ for 1 pass. The homogenization process decreased the size of the milk fat globules, dispersed them more uniformly, and improved the stability of 
the milk (Hayes and Kelly, 2003; Hayes et al., 2005). The homogenized milk sample was diluted to different concentrations with deionized water. The content of milk fat within diluted sample was measured using the Röse-Gottlieb method as well as dual-wavelength UV spectroscopy.

Diethyl ether (99.7\%), ethanol (99.7\%), n-heptane $(97 \%)$, and petroleum ether $\left(30-60^{\circ} \mathrm{C}\right)$ were of analytical reagent (AR) quality and were purchased from Sinopharm Chemical Reagent Co. Ltd. (Shanghai, China). Ammonia solution $(25 \sim 28 \%$, AR) was purchased from Guangdong Guanghua Sci-Tech Co., Ltd. (Guangzhou, China). Ethanol $(95 \%, \mathrm{AR})$ was purchased from Xilong Chemical Co., Ltd. (Shantou, China).

\section{Determination of Milk Fat Content Using the Röse-Gottlieb Method}

To determine the milk fat content using the RöseGottlieb method, a 5-mL milk sample was transferred into a 50-mL polypropylene screw-top centrifuge tube, followed by the addition of $0.63 \mathrm{~mL}$ of ammonia solution to adjust the $\mathrm{pH}$ value of the sample to about 9 . To release milk fat from fat globules, the mixture was heated in a water bath at $60^{\circ} \mathrm{C}$ for $5 \mathrm{~min}$. Subsequently, $5 \mathrm{~mL}$ of ethanol (95\%) was added into the mixture as de-emulsifier. The centrifuge tube was shaken vigorously to make sure that the emulsion was broken and the milk fat was released completely. The mixture with ethanol was then cooled to ambient temperature with tap water. Eight milliliters of diethyl ether and $8 \mathrm{~mL}$ of petroleum ether were added to extract the milk fat from the last mixture. The mixture containing diethyl ether and petroleum ether was vortexed for about $10 \mathrm{~s}$ and then centrifuged (Sorvall Biofuge Stratos, Thermo Electron LED GmbH, Osterode, Germany) at 4,000 × $g$ for $10 \mathrm{~min}$ at $25^{\circ} \mathrm{C}$ to separate the milk fat. After centrifugation, the supernatant was carefully transferred to an empty centrifuge tube. The above procedure was repeated to extract the remaining or trapped fat from the precipitate. The solvent content of the supernatant was allowed to evaporate at ambient temperature under a nitrogen atmosphere. Complete removal of organic solvents (i.e., diethyl ether and petroleum ether) from the milk fat was achieved by oven drying the sample at $70^{\circ} \mathrm{C}$. The drying processing was terminated until the difference in weights between 2 successive measurements was less than $1 \mathrm{mg}$. The milk fat content was determined by gravimetrically weighing the extracted fat using an analytical balance (AL204, Mettler-Toledo International Inc., Columbus, OH; Manirakiza et al., 2001).

\section{Determination of Milk Fat Content Using Dual-Wavelength UV Spectroscopy}

Preparation of Calibration Curve. Milk fat obtained using the Röse-Gottlieb method was used to prepare stock solution of milk fat. The stock solution of milk fat in $n$-heptane was diluted to different concentrations with $n$-heptane. Then, the diluted solution of milk fat was transferred to a cuvette with a $1-\mathrm{cm}$ path length. Ultraviolet light absorbance of the diluted solution was measured with a spectrophotometer (UV2550, Shimadzu Co., Kyoto, Japan) over a wavelength range of 200 to $300 \mathrm{~nm}$ at an interval of $0.1 \mathrm{~nm}$. The measurement was carried out at ambient temperature using $n$-heptane as reference. The measured absorbance was plotted against the milk fat content to construct the calibration curve.

Determination of Milk Fat Content. To determine the milk fat content in the diluted milk sample, $10 \mathrm{~mL}$ of the sample was transferred to a $50-\mathrm{mL}$ polypropylene screw-top centrifuge tube, followed by the addition of $14 \mathrm{~mL}$ of ethanol (99.9\%). The centrifuge tube with sample was vortexed for $30 \mathrm{~s}$ to break the emulsion and release milk fat from fat globules. After that, $10 \mathrm{~mL}$ of $n$-heptane was added to the centrifuge tube. To facilitate the extraction of milk fat with $n$ heptane, the centrifuge tube containing the sample was vortexed again for $30 \mathrm{~s}$ and left to stand for $10 \mathrm{~min}$ at ambient temperature, during which a biphasic system was observed. The biphasic system was centrifuged at $4,000 \times g$ for $5 \mathrm{~min}$ at $25^{\circ} \mathrm{C}$ and then left to stand for $1.5 \mathrm{~h}$ at ambient temperature to separate the milk fat completely. The supernatant obtained in this way was transferred to a cuvette with a $1-\mathrm{cm}$ path length. The UV light absorbance of the supernatant was measured using the procedure described above. The supernatant of a mixture containing $10 \mathrm{~mL}$ of deionized water, 14 $\mathrm{mL}$ of ethanol $(99.9 \%)$, and $10 \mathrm{~mL}$ of $n$-heptane was used as a reference. The fat content in diluted milk sample was calculated from the measured absorbance value, referring to the calibration curve described above. The procedure to determine milk fat content is shown schematically in Figure 1. In the case of a milk sample containing a relatively high percentage of fat, repeated extraction of fat was performed.

\section{Statistical Analysis}

All the experiments were carried out in triplicate. The results reported are means \pm standard deviations of parallel determinations. One-way ANOVA was performed to compare means of parallel determinations. A value of $P<0.05$ was considered significant. 

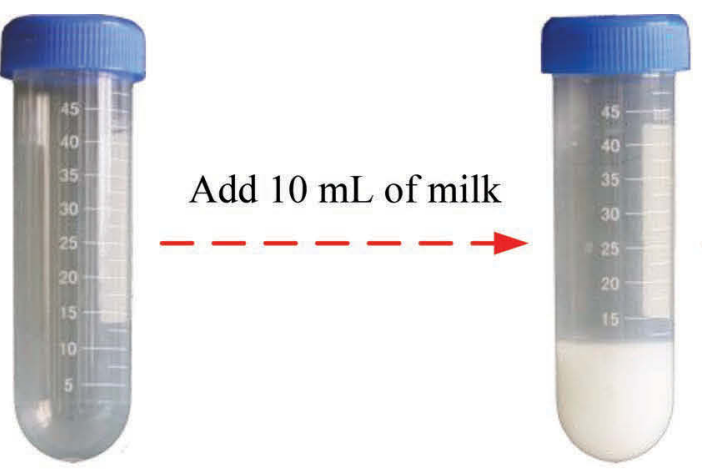

Add $14 \mathrm{~mL}$ of ethanol

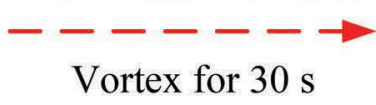

Vortex for $30 \mathrm{~s}$

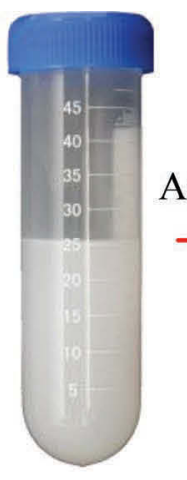

Add $10 \mathrm{~mL}$ of $\mathrm{n}$-heptane

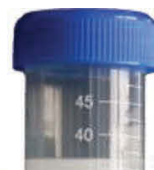

Vortex for $30 \mathrm{~s}$ Stand for $10 \mathrm{~min}$

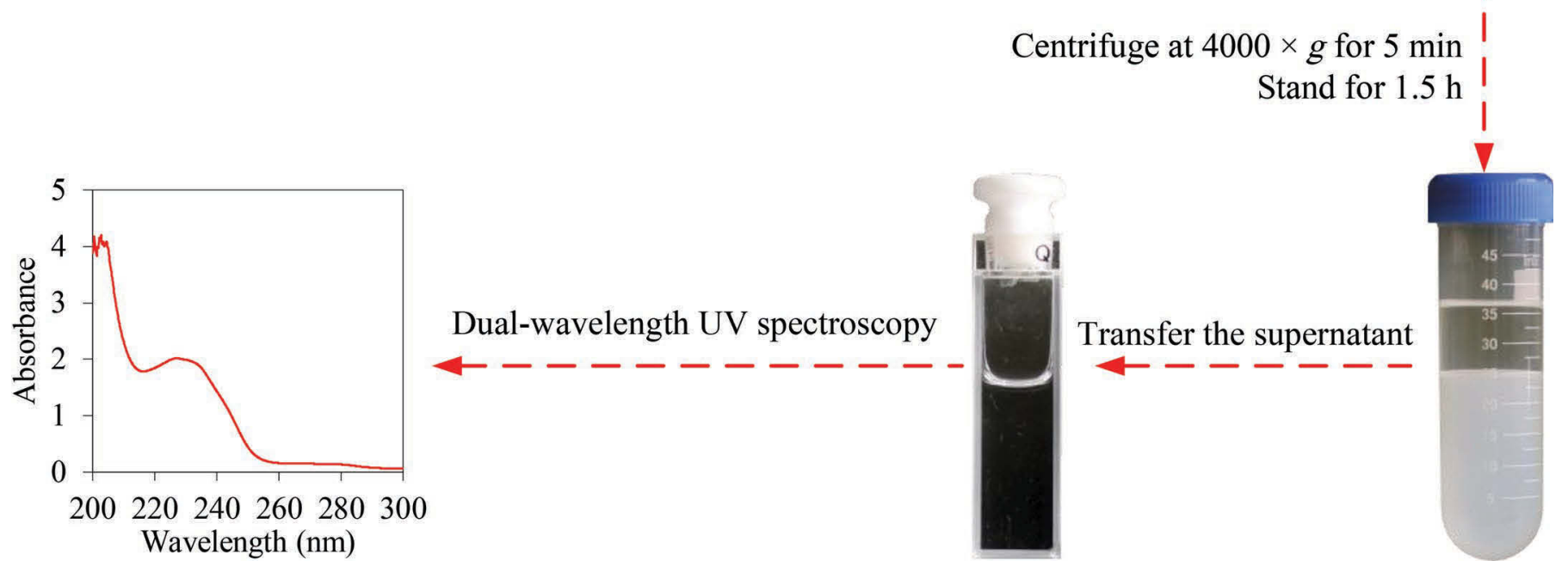

Figure 1. The procedure to determine ultra-low content of milk fat using dual-wavelength UV spectroscopy. Color version available online.

\section{RESULTS AND DISCUSSION}

\section{UV Spectra of Milk Fat}

Milk fat contains certain amount of conjugated fatty acid, which is quite different from other natural fats and oils. Typical UV spectra of milk fat recorded are shown in Figure 2. Milk fat absorbs UV light at about 205, 230, and $270 \mathrm{~nm}$. The absorption at $205 \mathrm{~nm}$ is due to the presence of double bonds in the fatty acyl groups (Gunstone et al., 1994), whereas the absorption at 230 and $270 \mathrm{~nm}$ is due to conjugated dienes and trienes of UFA, respectively (Kapoor et al., 2005). As expected, the extent of absorbance increased with the increase in the milk fat content. This observation shows that it is possible to determine the milk fat content in diluted milk sample using UV spectroscopy.

\section{Calibration Curve of Dual-Wavelength UV Spectroscopy}

As the milk fat absorbs UV light at both 230 and 270 $\mathrm{nm}$, the difference in absorbance at these 2 wavelengths was plotted against the milk fat content to construct the calibration curve. As shown in Figure 3, the increase in milk fat content was linear in absorbance values obtained at 230 and $270 \mathrm{~nm}$. The linear correlation covered a wide range of content of milk fat, which means that milk with widely different fat contents can be determined using UV spectroscopy. The lowest milk fat content that could be determined accurately using this method was about $140 \mathrm{mg} / \mathrm{L}$. This is significantly lower than the milk fat content that can be measured using the Röse-Gottlieb method; thus, the UV spectroscopic method is much more accurate and can measure much lower fat content in milk.

The difference between the current work and that of Forcato et al. (2005) is that those authors did not use an organic solvent to extract milk fat. Milk fat is poorly soluble in a water-ethanol mixture; thus, the absorbance in their work was relatively low, especially at the characteristic wavelengths of 230 and $270 \mathrm{~nm}$. This is probably why they did not use the absorbance of milk fat at 230 and $270 \mathrm{~nm}$, but instead measured absorbance at 208,210,212, and $215 \mathrm{~nm}$ to construct the calibration curve. The extent of UV absorbance of 


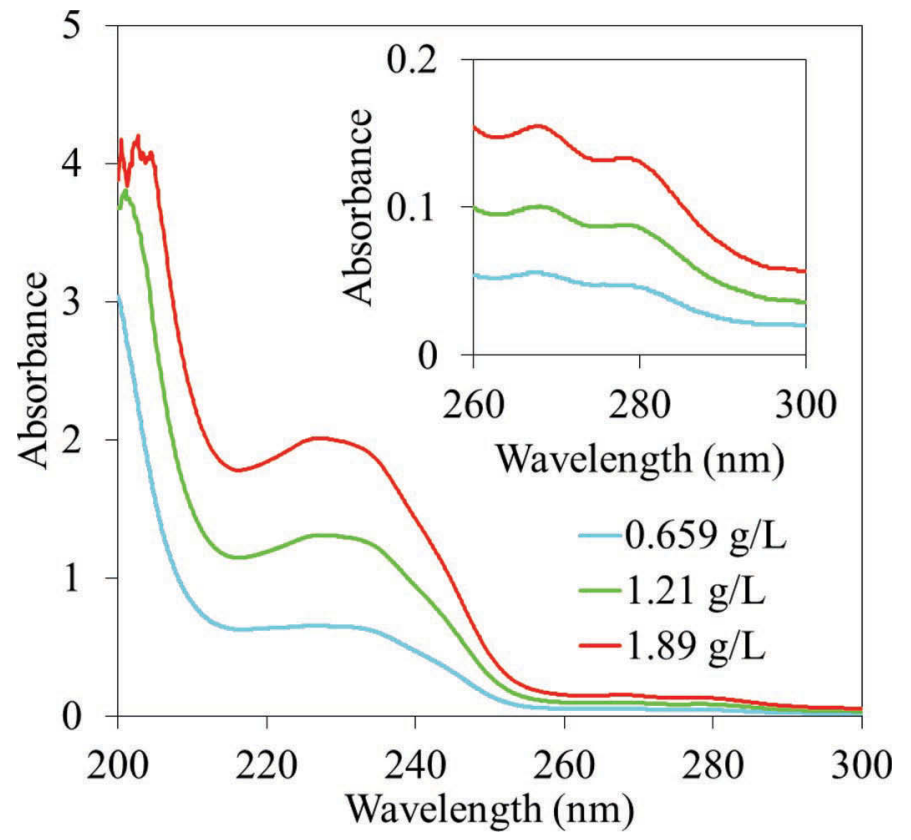

Figure 2. Ultraviolet spectra of milk fat with different contents in $n$-heptane. Insert shows the spectra from 260 to $300 \mathrm{~nm}$. Color version available online.

milk fat at 208, 210, 212, and $215 \mathrm{~nm}$ was also studied in the current work. As shown in Figure 4, the absorbance of milk fat at 208, 210,212, and $215 \mathrm{~nm}$ increased

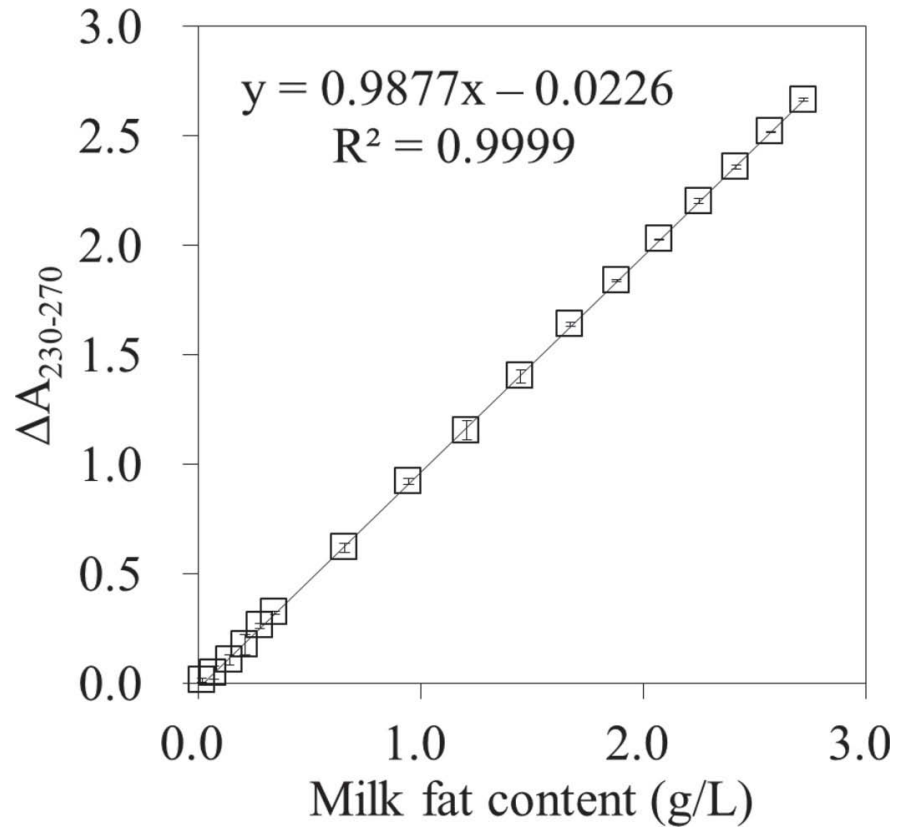

Figure 3. The difference in UV light absorbance $(\Delta \mathrm{A})$ of milk fat at 230 and $270 \mathrm{~nm}$ plotted against the milk fat content. The error bars indicate the SD of 3 parallel determinations.

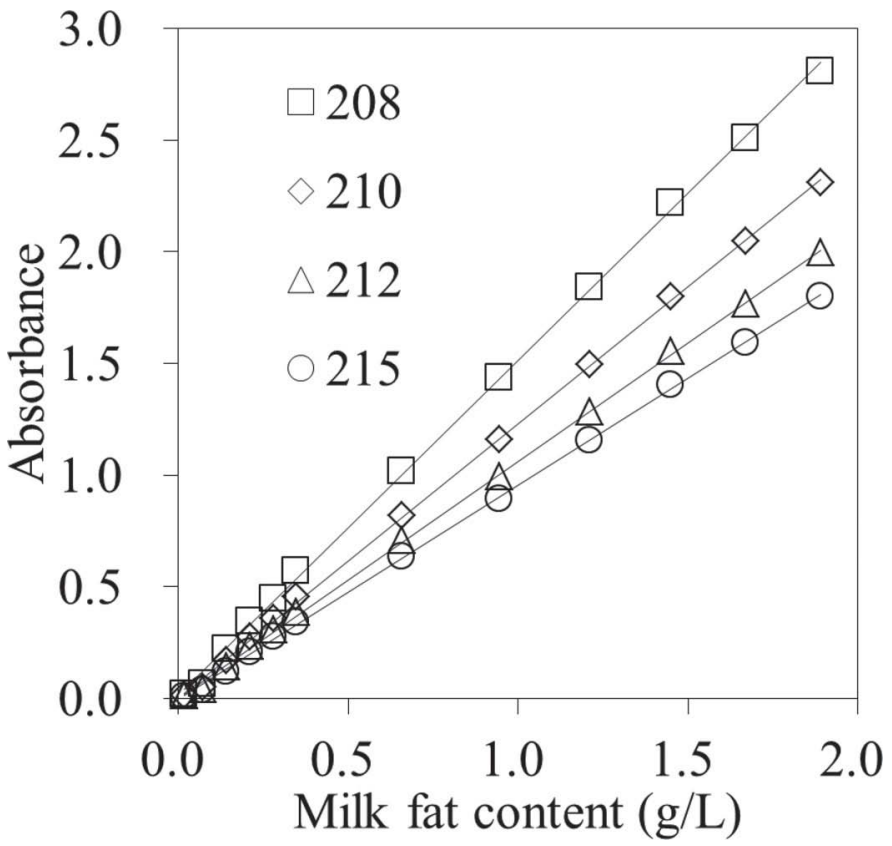

Figure 4. The UV light absorbance of milk fat at 208, 210, 212, and $215 \mathrm{~nm}$ plotted against the milk fat content.

linearly as the milk fat content increased. However, the absorbance of UV by milk fat at these wavelengths is sensitive to the change in UV wavelength, which might result in an unexpected error in measured value of milk fat. Thus, the absorbance of milk fat at 208, 210, 212, and $215 \mathrm{~nm}$ was not adopted in the present work.

\section{Precision of Dual-Wavelength UV Spectroscopy}

The milk fat content was measured using both RöseGottlieb method and dual-wavelength UV spectroscopy and the results are listed in Table 1 for comparison. In whole milk, the fat content measured using the RöseGottlieb method was $37.0 \mathrm{~g} / \mathrm{L}$, which agrees well with the value indicated on the package. However, the measured fat content deviated significantly from the known value as the fat content decreased. The increasing error of determination indicated that the Röse-Gottlieb method was not suitable to determine fat content at low concentrations.

As shown in Table 1, the content of milk fat determined by the dual-wavelength UV spectroscopy agreed well with the value calculated (i.e., the fat content of whole milk divided by the dilution factor). The error of determination was less than $6 \%$. The standard deviation of 3 parallel determinations was 0.03 or less. The standard deviation expressed as a percentage of the mean value (i.e., the relative standard deviation) was less than $7 \%$, indicating that the method developed in 
Table 1. Comparison of milk fat contents determined by the dual-wavelength UV spectroscopy and the RöseGottlieb method

\begin{tabular}{|c|c|c|c|c|}
\hline \multirow{2}{*}{$\begin{array}{l}\text { Milk fat } \\
\text { content }(\mathrm{g} / \mathrm{L})\end{array}$} & \multicolumn{2}{|c|}{ UV spectroscopy } & \multicolumn{2}{|c|}{ Röse-Gottlieb method } \\
\hline & Mean $\pm \mathrm{SD}(\mathrm{g} / \mathrm{L})$ & Error $(\%)$ & Mean $\pm \mathrm{SD}(\mathrm{g} / \mathrm{L})$ & Error (\%) \\
\hline 37.0 & - & - & $37.0 \pm 0.5$ & 0 \\
\hline 1.76 & $1.70 \pm 0.03$ & -4 & $1.7 \pm 0.4$ & -4 \\
\hline 0.725 & $0.709 \pm 0.007$ & -2.1 & $0.31 \pm 0.26$ & -57 \\
\hline 0.366 & $0.357 \pm 0.008$ & -2 & - & - \\
\hline 0.147 & $0.156 \pm 0.010$ & 6 & - & - \\
\hline
\end{tabular}

this work to determine ultra-low content of milk fat is reproducible.

It should be noted that the fat content in commercial dairy products is relatively high; for example, whole milk normally has a fat content $\geq 37 \mathrm{~g} / \mathrm{L}$. The fat content in whole milk cannot be determined directly using the procedure mentioned above, because the absorbance of milk fat is beyond the linearity range of calibration curve. An alternative way to determine the fat content in whole milk is to dilute the sample with deionized water before the test.

The most frequently used organic solvent to extract milk fat is $n$-heptane. However, the presence of ethanol facilitates the dissolution of milk fat in water, which alters the partition coefficient of milk fat in $n$-heptane or water system (Bravo et al., 2008). Repeated extraction of milk fat with $n$-heptane is thus necessary for milk with high fat content. For the milk sample with a fat content of $1.76 \mathrm{~g} / \mathrm{L}$, only $87.0 \%$ of the milk fat was extracted in $n$-heptane in the first extraction, whereas $96.7 \%$ of milk fat was extracted in the second extraction.

\section{Spike Recovery of the Dual-Wavelength UV Spectroscopy}

To assess the accuracy of dual-wavelength UV spectroscopy presented in this work, we performed a spike recovery assay. Milk fat solution in $n$-heptane with a known fat content was used as the spike. A specified amount of spike was added directly to the diluted milk sample with a known content of fat. The amount of fat in spiked milk was determined in the same manner as was done in unknowns. The amount of added milk fat was calculated by subtracting the known amount of fat in diluted milk sample from that determined in spiked milk. Percentage of the spike recovery of milk fat is the ratio of the value determined to its known value.

The percentage of the spike recovery is listed in Table 2 . The mean percentage spike recovery varied from 97 to $103 \%$, with a maximum standard deviation of $10 \%$, which is acceptable for the determination of milk fat (Forcato et al., 2005).

\section{CONCLUSIONS}

We present a new approach to determine ultra-low fat content in milk, which consists of the extraction of milk fat with $n$-heptane and subsequent determination of extracted milk fat using dual-wavelength UV spectroscopy. Milk fat in the globule is released by adding ethanol followed by the extraction with $n$-heptane. This method makes use of the unique UV absorption properties of milk fat at 230 and $270 \mathrm{~nm}$, which is directly proportional to the fat content. The results showed that milk fat could be extracted completely and quantified accurately using this new approach. The reproducibility, error, and spike recovery of determination were all within acceptable ranges. As the calibration curve is not plotted against the milk fat content determined by any reference method, the accuracy of the proposed dual-wavelength UV spectroscopic method does not

Table 2. Spike recovery of milk fat

\begin{tabular}{lrccc}
\hline & \multicolumn{4}{c}{ Spike recovery (\%) } \\
\cline { 2 - 5 } $\begin{array}{l}\text { Milk fat } \\
\text { content }(\mathrm{g} / \mathrm{L})\end{array}$ & Run 1 & Run 2 & Run 3 & Mean $\pm \mathrm{SD}^{1}$ \\
\hline 1.76 & 98 & 100 & 96 & $98 \pm 2$ \\
0.725 & 95 & 108 & 92 & $98 \pm 8$ \\
0.366 & 106 & 99 & 87 & $97 \pm 10$ \\
0.147 & 98 & 110 & 101 & $103 \pm 7$ \\
\hline
\end{tabular}

${ }^{1}$ Values in this column do not differ significantly at $P<0.05$ level. 
rely on any reference method. Another advantage of this method is that only a very small amount of organic solvent is required to extract the milk fat completely.

\section{ACKNOWLEDGMENTS}

The work was supported by the Fundamental Research Funds for the Central Universities of P. R. China (20720150092).

\section{REFERENCES}

Badertscher, R., T. Berger, and R. Kuhn. 2007. Densitometric determination of the fat content of milk and milk products. Int. Dairy J. $17: 20-23$.

Bernuy, B., M. Meurens, E. Mignolet, and Y. Larondelle. 2008. Performance comparison of UV and FT-Raman spectroscopy in the determination of conjugated linoleic acids in cow milk fat. J. Agric. Food Chem. 56:1159-1163.

Bravo, B., J. Sánchez, A. Cáceres, G. Chávez, F. Ysambertt, N Márquez, M. Jaimes, M. I. Briceño, and J. L. Salager. 2008. Partitioning of fatty acids in oil/water systems analyzed by HPLC. J. Surfactants Deterg. 11:13-19.

Carl, R. T. 1991. Quantification of the fat content of milk using a partial-least-squares method of data analysis in the near infrared. Fresenius J. Anal. Chem. 339:70-71.

El-Abassy, R. M., P. J. Eravuchira, P. Donfack, B. von der Kammer, and A. Materny. 2011. Fast determination of milk fat content using Raman spectroscopy. Vib. Spectrosc. 56:3-8.

Forcato, D. O., M. P. Carmine, G. E. Echeverría, R. P. Pécora, and S. C. Kivatinitz. 2005. Milk fat content measurement by a simple UV spectrophotometric method: an alternative screening method. J. Dairy Sci. 88:478-481.
Fox, P. F. 2011. Encyclopedia of Dairy Sciences. 2nd ed. Academic Press, San Diego, CA.

Gunstone, F. D., J. L. Hardwood, and F. B. Padley. 1994. The Lipid Handbook. 2nd ed. Chapman \& Hall, London, UK.

Hayes, M. G., P. F. Fox, and A. L. Kelly. 2005. Potential applications of high pressure homogenisation in processing of liquid milk. J. Dairy Res. 72:25-33.

Hayes, M. G., and A. L. Kelly. 2003. High pressure homogenization of raw whole bovine milk effects on fat globule size and other properties. J. Dairy Res. 70:297-305.

James, C. S. 1995. Analytical Chemistry of Foods. Chapman \& Hall, London, UK.

Kapoor, R., M. Reaney, and N. D. Westcott. 2005. Bailey's Industrial Oil and Fat Products. 6th ed. John Wiley \& Sons Inc., Hoboken, NJ.

Manirakiza, P., A. Covaci, and P. Schepens. 2001. Comparative study on total lipid determination using Soxhlet, Roese-Gottlieb, Bligh \& Dyer, and modified Bligh \& Dyer extraction methods. J. Food Compos. Anal. 14:93-100.

Milk Facts. 2015. Milk Composition-Milk Fat. Accessed Dec. 31 , 2015. http://www.milkfacts.info/Milk\%20Composition/Fat.htm.

Povolo, M., and G. Contarini. 2009. Fast gas chromatography: Applications in milk fat analysis. Lipid Technol. 21:88-90.

Spanos, G. A., S. J. Schwartz, R. B. van Breemen, and C.-H. Huang. 1995. High-performance liquid chromatography with light-scattering detection and desorption chemical-ionization tandem mass spectrometry of milk fat triacylglycerols. Lipids 30:85-90.

Tsenkova, R., S. Atanassova, K. Toyoda, Y. Ozaki, K. Itoh, and T. Fearn. 1999. Near-infrared spectroscopy for dairy management: Measurement of unhomogenized milk composition. J. Dairy Sci. $82: 2344-2351$

Xin, Q., H. Z. Ling, T. J. Long, and Y. Zhu. 2006. The rapid determination of fat and protein content in fresh raw milk using the laser light scattering technology. Opt. Lasers Eng. 44:858-869.

Zhu, X., W. Guo, and Z. Liang. 2015. Determination of the fat content in cow's milk based on dielectric properties. Food Bioproc. Techol. $8: 1485-1494$. 